\title{
SCIENTIFIC REPORTS

\section{Association between medicated obstructive pulmonary disease, depression and subjective health: results from the population-based Gutenberg Health Study}

Jasmin Ghaemi Kerahrodi ${ }^{1 *}$, Elmar Brähler ${ }^{1}$, Jörg Wiltink ${ }^{1}$, Matthias Michal ${ }^{1}$, Andreas Schulz ${ }^{3}$, Phillip S. Wild $\mathbb{D}^{3,4,5}$, Thomas Münzel ${ }^{2,5}$, Gerrit Toenges ${ }^{7}$, Karl J. Lackner ${ }^{6,5}$, Norbert Pfeiffer ${ }^{8}$ \& Manfred E. Beutel ${ }^{1}$

Medicated obstructive pulmonary disease (asthma or COPD) has been associated with depression. Yet, there is little knowledge of the interplay of contributing social, biological, behavioral and psychological factors in the community. The study was conducted: (1) To determine the prevalence of depression in participants with medicated COPD or asthma from the general population, (2) to identify underlying social, biological, behavioral and psychological factors and (3) to determine the contribution of obstructive pulmonary disease and depression to subjective health. The population-based sample of 15.010 study participants (35-74 years) from the Gutenberg Health Study (GHS) was queried according to a medical diagnosis of obstructive pulmonary disease, defined as medicated COPD or asthma, and comorbid disorders. Demographic, behavioral and psychological factors were assessed by selfreport; lung function (FEV1; FCV) was measured by spirometry. 307 men (4.3\%) and 396 women (5.6\%) reported a medical diagnosis of COPD or asthma. The prevalence of depression (PHQ-9 > $=10$ ) was twice as high ( $16.2 \%$ vs. $7.5 \%)$ compared to participants without obstructive pulmonary disease. Participants with obstructive pulmonary disease were older, had a lower SES, more comorbid diseases and cardiovascular risk factors, higher distress and took more psychotropic medication. In multivariable logistic regression analyses, obstructive pulmonary disease was associated with a $71 \%$ increase of depression $(\mathrm{OR}=1.71 ; 95 \% \mathrm{Cl}=1.30$ to 2.24$)$. Additional contributors were $\mathrm{FEV} 1(1.18 ; 95 \% \mathrm{Cl}=1.05$ to 1.32 ) and dyspnea (NYHA $>=1)(2.19 ; 1.82$ to 2.64$)$, sex (women) (OR=1.73; $95 \% \mathrm{Cl} 1.41$ to 2.12 ), lower SES (OR $=0.98 ; 95 \% \mathrm{Cl}=0.96$ to 0.99$)$. Lack of active sports $\mathrm{OR}=0.79 ; 95 \% \mathrm{Cl} 0.68$ to 0.92$)$, obesity (OR 1.27; $95 \% \mathrm{Cl} 1.07$ to 1.50 ), smoking (OR $=1.26 ; 95 \% \mathrm{Cl} 1.06$ to 1.49 ) and dyslipidemia $(\mathrm{OR}=1.35 ; 95 \% \mathrm{Cl} 1.15$ to 1.57$)$ also increased the risk of depression. Additional psychological risks were social phobia, type $D$, low social support, loneliness and life events in the past 12 months. In multivariable linear regression analyses, obstructive pulmonary disease and depression independently contributed to reduced subjective health in addition to sedentary behavior, smoking and comorbid somatic and mental disorders. These findings provide evidence that COPD and asthma are associated with depression in the community. Complex underlying demographic, medical and psychosocial

${ }^{1}$ Department of Psychosomatic Medicine and Psychotherapy, University Medical Center of the Johannes Gutenberg University Mainz, Mainz, Germany. ${ }^{2}$ Center for Cardiology, Cardiology I, University Medical Center of the Johannes Gutenberg University Mainz, Mainz, Germany. ${ }^{3}$ Preventive Cardiology and Preventive Medicine, Center for Cardiology, University Medical Center of the Johannes Gutenberg-University Mainz, Mainz, Germany. ${ }^{4}$ Center for Thrombosis and Hemostasis, University Medical Center of the Johannes Gutenberg-University Mainz, Mainz, Germany. ${ }^{5}$ DZHK (German Center for Cardiovascular Research), partner site RhineMain, Mainz, Germany. ${ }^{6}$ Institute for Clinical Chemistry and Laboratory Medicine, University Medical Center of the Johannes Gutenberg University Mainz, Mainz, Germany. ${ }^{7}$ Institute for Medical Biostatistics, Epidemiology and Informatics, University Medical Center of the Johannes Gutenberg-University Mainz, Mainz, Germany. ${ }^{8}$ Department of Ophthalmology, University Medical Center of the Johannes Gutenberg-University Mainz, Mainz, Germany. *email: jasmin.ghaemi@unimedizin-mainz.de 
variables have been identified which may justify an integrative treatment approach. Promoting health behavior (smoking cessation, exercising, weight reduction) and social integration may not only improve the somatic course of the disease, but also mental health. Mental health treatment may also improve health behavior and subjective health.

Chronic obstructive pulmonary disease (COPD) is a major cause of worldwide morbidity and mortality. It is a complex, heterogeneous and multicomponent disease, characterized by airflow limitation, chronic systemic inflammation and progressing fibrosis with remodeling and narrowing of the small airways and destruction of the lung parenchyma ${ }^{1}$. Multiple somatic, esp. cardiovascular and mental comorbidities have been described ${ }^{2,3}$ affecting clinical severity and prognosis ${ }^{4,5}$. Risk factors overlap between COPD and other diseases, especially cardiovascular risk factors $\mathrm{CVRFs}^{5}$, most prominent is smoking ${ }^{6}$.

While depression has been considered one of the most frequent comorbidities in COPD, prevalence estimates have ranged from $18.8 \%{ }^{7}$ to $51.5 \%{ }^{8}$. Somatic (cardiovascular, diabetes) and mental comorbidities (depression, anxiety) were negatively associated with health-related quality of life (HRQOL) and health outcomes in patients with COPD ${ }^{9-11}$, increased health resource use ${ }^{12}$ and mortality ${ }^{10,13,14}$.

Associations of asthma with psychological factors have been investigated. Individuals with asthma have reported reduced health-related quality of life ${ }^{15}$. There is a wide variation of the prevalence of depressive symptoms in individuals with asthma ranging from $1 \%$ to $45 \%{ }^{16}$.

Dyspnea is one of the most common symptoms in pulmonary and cardiovascular medicine. Patients with chronic diseases that lead to impaired lung function and dyspnea, are likely to have more depressive symptoms compared with healthy individuals ${ }^{17}$. Most likely, mechanisms work both ways ${ }^{13}$ : obstructive pulmonary diseases such as COPD and asthma increase the risk for mental disorders ${ }^{18}$ for biological (dyspnea, chronic inflammation) and psychosocial reasons. On the other hand, mental distress increases the risk of smoking, the primary risk factor for COPD ${ }^{6,19}$. Conversely, smoking has been shown a risk factor for new onset of depression in middle-aged adults $^{20}$. Also, there may be an influence of emotional states on lung function. Negative mood states were associated with a reduction in FEV1 compared with neutral states of emotion ${ }^{21}$. Ritz et al..$^{22}$ found an increased total respiratory resistance in subjects with asthma following exposure to depressing stimuli.

In a comprehensive approach we analyzed the relationships ${ }^{3}$ of social, biological, and psychological variables with COPD or asthma and depression in a large community sample. Thus, analyses covered participants who reported that they were diagnosed and treated with COPD or asthma by a physician (and not limited to those who seek health care as in most previous studies). Yet, we were able to include a comprehensive range of biological variables including pulmonary function, symptoms (dyspnea) and comorbid disorders, social and psychological variables. Aims of the study were 1): To determine the prevalence of depression in participants with medicated COPD or asthma in the general population, and 2) to identify underlying associations of social, biological, behavioral and psychological factors and 3) to determine the contribution of obstructive pulmonary disease and depression to subjective health.

We expected an increased prevalence of depression among participants with obstructive pulmonary disease (COPD or Asthma) and a higher proportion of depression in participants with a) worse lung function as evidenced by FEV1 and dyspnea, b) additional somatic disease (CVD, cancer, etc.), c) stress (life events, loneliness) and Type D behavior. We further expected lower subjective health in participants suffering from both COPD or asthma and depressive symptoms.

\section{Material and Methods}

Participants and recruitment. The analysis presents data of a large-scale population-based sample. 15.010 study participants were enrolled in the Gutenberg Health Study (GHS) between the years of 2007 and 2012. The prospective, population-based, observational single-center cohort study of the University Medical Center Mainz is conducted in the Rhine-Main-Region of Western Germany. The design and the rationale of the Gutenberg Health Study (GHS) have been described in detail elsewhere ${ }^{23}$. This report is based on cross-sectional analy$\mathrm{ses}^{24}$. Participants were randomly drawn from the local registry of the city of Mainz and the county of MainzBingen. The study protocol and documents were approved by the ethics committee of the Medical Chamber of Rhineland-Palatinate and the local data safety commissioner. All study investigations have been conducted in line with the Declaration of Helsinki and principles outlined in recommendations for Good Clinical Practice and Good Epidemiological Practice. Accordingly, written informed consent was obtained from all participants prior to entering the study. Inclusion criterion was age between 35 and 75 years. Exclusion criteria were insufficient knowledge of German language and inability to participate due to physical or mental impairment. The sample was stratified 1:1 for gender and residence (rural and urban) and in equal strata for decades of age. Out of a sample of $28,533,52.61 \%$ participated $(\mathrm{N}=15,010 ; 53.3 \%$ of the men and $51.9 \%$ of the women). Five point two percent fulfilling exclusion criteria were excluded. Of the non-responders, $22.6 \%$ refused participation, and another $20 \%$ could not be contacted.

Computer-assisted personal interview. Prevalent cardiovascular risk factors and other clinical variables were collected by a computer-assisted personal interview during the 5-hour baseline-examination in the study center. Blood pressure, anthropometric measurements and laboratory examinations from a venous blood sample were performed by certified medical technical assistants according to standard operating procedures.

The widely used Patient Health Questionnaire (PHQ-9) was used for screening the presence and severity of depressive disorder. It has been proven a reliable measure of depression ${ }^{25}$. The PHQ-9 scores each of the 9 DSM-IV criteria as "0" (not at all), "1" (several days), " 2 " (more than half of the days) and " 3 " (nearly every day). 
A PHQ-9 sum score $\geq 10$ was defined as probable caseness. Lowe et al. found a sensitivity of $81 \%$ and a specificity of $82 \%$ for depressive disorder determined by this cut-off ${ }^{25}$.

Suicidal thoughts were measured by the single item (PHQ-9) "in the last two weeks, have you had thoughts that you would be better off dead or hurting yourself in some way?”.

We assessed generalized anxiety with the two item short form of the GAD-7 (Generalized Anxiety Disorder [GAD] - 7 Scale). Participants rated the items "feeling nervous, anxious or on edge" and "not being able to stop or control worrying" on the same scale as the PHQ-9. A sum score of 3 and more (range 0-6) indicated generalized anxiety with good sensitivity $(86 \%)$ and specificity $(83 \%)^{26}$.

The brief PHQ panic module was used to screen panic disorder. If at least two of the first four panic questions were answered with "yes", caseness was defined ${ }^{27}$.

Social phobia was assessed with the German version of the Mini-Social Phobia Inventory (Mini-Spin) ${ }^{28}$ (e.g. "I avoid activities which put me in the center of attention"), rated from "not at all" $(=0)$ to "extremely" $(=3)$. Based on the cut-off score of 6 (range $0-12$ ), the Mini-Spin has a good sensitivity (89\%) and specificity (90\%) to detect individuals with social anxiety disorder ${ }^{28}$.

Type D Personality was assessed with the German version of the DS- $14^{29}$ with two 7 -item subscales, negative affectivity (NA) and social inhibition (SI). It is answered on a 5-point- Likert scale from 0 (false) to 4 (true). The internal consistency of the DS-14 subscales is high (NA Cronbach's $\alpha=0.87$, SI Cronbach's $\alpha=0.86$ ). Type D personality is defined as a pattern of significant negative affectivity (NA $\geq 10)$ in conjunction with significant social inhibition (SI $\geq 10)$.

Socioeconomic status (SES) was defined according to Lampert et al..$^{30}$ from 3 (lowest) to 21 (highest socioeconomic status) based on education, profession and income. Income was additionally calculated according to OECD.

Loneliness was assessed with the single item "I am frequently alone /have few contacts". The item rated from $0=$ "no, does not apply", 1 = "yes it applies, but I do not suffer from it", 2 = "yes, it applies, and I suffer slightly", $3=$ "yes, it applies, and I suffer moderately", to $4=$ "yes, it applies, and I suffer strongly". Loneliness was defined combining 0 and $1=$ no loneliness or distress; $2=$ slight, $3=$ moderate, and $4=$ severe loneliness ${ }^{31}$.

Subjective health status was assessed by the question: "How would you describe your current health status?" $(0=$ worst $; 100=$ best health status $)$.

Alcohol consumption and abuse was measured in gram per day; alcohol abuse was defined as daily consumption $\geq 40 \mathrm{mg}$ for women and $\geq 60 \mathrm{mg}$ for men.

The Short Questionnaire to Assess Health-Enhancing Physical Activity (SQUASH) evaluated physical activity $^{32}$. It captures household, commuting, leisure time, work and school activities. Standing, lying, sleeping and sitting were classified as inactivity. Active sports were rated as the highest quartile of physical activity.

The presence of cardiovascular disease (CVD) was defined as history of self-reported myocardial infarction (MI), heart failure (HF), stroke, deep vein thrombosis (DVT), pulmonary embolism (PE), and peripheral arterial disease (PAD). Coronary heart disease was assessed by the question: "Were you diagnosed with a stenosis of your coronary vessels?" Cancer, COPD and asthma were assessed, correspondingly. Further, participants were asked, whether they had ever received the definite diagnosis of depressive disorder or any anxiety disorder by a physician.

Smoking was dichotomized into non-smoker and smoker. Non-smokers were defined as never and ex-smokers. Smokers were defined as occasional, i.e. $<1$ cigarette/day and regular smokers, i.e. $>1$ cigarette/day. Weight and height were measured according to a standardized, written manual. Obesity was defined as a body mass index $(\mathrm{BMI}) \geq 30 \mathrm{~kg} / \mathrm{m}^{2}$. BMI was calculated (weight in $\mathrm{kg}$ divided by the square of height in meters) ${ }^{33}$. Diabetes was based on a blood glucose level of $\geq 126 \mathrm{mg} / \mathrm{dl}$ in the baseline examination after an overnight fast of at least $8 \mathrm{~h}$, respectively a blood glucose level of $\geq 200 \mathrm{mg} / \mathrm{dl}$ after a fasting period $<8 \mathrm{~h}$ or the diagnosis of diabetes by a physician. Dyslipidemia was defined as a low-density lipoprotein/high-density lipoprotein ratio of $>3.5$ or a diagnosis by a physician. Hypertension was diagnosed if the mean systolic blood pressure was $\geq 140 \mathrm{~mm} \mathrm{Hg}$ (diastolic $\geq 90 \mathrm{~mm} \mathrm{Hg}$ ) in the $2 \mathrm{nd}$ and $3 \mathrm{rd}$ standardized measurement after 8 and $11 \mathrm{~min}$ of rest or if antihypertensive drugs were taken. FEV1 and FCV were assessed by spirometry ${ }^{34}$.

The New York Heart Association (NYHA) Functional Classification measures how much participants are limited during physical activity. NYHA 1 was defined as no symptoms and no limitation in ordinary physical activity, e.g. no shortness of breath when walking, climbing stairs, NYHA 2 was defined as mild symptoms (mild shortness of breath and/or angina) and slight limitation during ordinary activity.

Medications were registered at the study center by scanning the bar codes from the original drug packages of the participants. Three classes of antidepressants were entered as dichotomous variables (yes/no) as described by the ATC code: selective serotonin reuptake inhibitors (ATC N06AB), non-selective monoamine reuptake inhibitors (ATC N06AA) and other antidepressants (ATC N06AX).

Statistical analysis. Descriptive analysis were performed as absolute and relative proportions for categorical data, means and standard deviations for continuous variables and median with interquartile range if not fulfilling normal distribution. Inference tests between depression groups (no/yes) were calculated with t-tests or Chi2 tests.

To analyze the relationship between depression and COPD, we used logistic regression analysis on the presence of depression (PHQ-9 $>$ or $<10$ ).

To determine relations between depression, COPD and subjective health we used stepwise linear regression models. We also used stepwise linear regression models for men and women separately In the first model we included all sociodemographic, behavioral and psychological variables. In the second model we additionally included major somatic disorders (diabetes, CVD, COPD, cancer) in order to determine their additional 
contribution to somatic symptoms when the sociodemographic, behavioral and psychological variables are also in the equation.

All p-values should be regarded as a continuous parameter that reflect the level of statistical evidence and are therefore reported exactly. In addition, p-values are not adjusted for multiple testing. Hence all of the analyses are exploratory. Statistical analysis was carried out using R version 3.3.1.

\section{Results}

Sample characteristics. A total of $4.9 \%$ of the participants suffered from COPD or Asthma. Table 1 (baseline table) shows psychosocial and medical characteristics of participants with obstructive pulmonary disease compared to those without obstructive pulmonary disease.

Participants suffering from obstructive pulmonary disease were older and had a lower SES. They had clearly impaired lung function (FEV1, FVC), and almost half of the sample suffered from at least mild dypnea. There was an increased rate of cardiovascular risk factors, including obesity, smoking, a lack of active sports, hypertension, dyslipidemia and an increase of cardiovascular disease, diabetes, cancer and family history of MI, resp. stroke. While the great majority lived in a partnership, social support was reduced, and they reported more loneliness and type $\mathrm{D}$ behavior. There was no difference regarding social phobia.

As Table 1 shows, participants with obstructive pulmonary disease were older, more often female and had a lower SES. Mean pulmonary function was reduced based on FEV1 and FVC, and almost half suffered at least from mild dyspnea (13\% from moderate to severe dyspnea). Obstructive pulmonary disease was associated with obesity, diabetes, slightly more current smoking (22.8\% vs. $19.4 \%)$, less active sports and alcohol consumption, and more hypertension and dyslipidemia. There was also a high comorbidity with CVD including myocardial infarction (MI), stroke and cancer and a family history of MI, resp. stroke.

As psychosocial vulnerability factors, participants with obstructive pulmonary disease had lower SES, income and employment, and suffered from more loneliness and lower subjective health. As a psychobiological vulnerability factor, they had an increased rate of type $\mathrm{D}$ personality. They were more likely to have somatic comorbidities and more CVRFs compared to participants without COPD.

Table 2 shows multivariable logistic regression analyses with depression as dependent variable for all participants.

In multivariable logistic regression analyses, obstructive pulmonary disease was associated with increased depression $(\mathrm{OR}=1.71 ; 95 \% \mathrm{CI}=1.30$ to 2.24$)$. Additional predictors were medical: $\mathrm{FEV} 1(1.18 ; 95 \% \mathrm{CI}=1.05$ to 1.32$)$ and dyspnea (NYHA $>=1)(\mathrm{OR}=2.19 ; \mathrm{CI}=1.82$ to 2.64$)$, demographic: sex (women) $(\mathrm{OR}=1.73$; $95 \% \mathrm{CI}=1.41$ to 2.12 ), lower $\mathrm{SES}(\mathrm{OR}=0.98 ; 95 \% \mathrm{CI}=0.96$ to 0.998$)$. Cardiovascular risk factors such as active sports $(\mathrm{OR}=0.79 ; 95 \% \mathrm{CI}=0.68$ to 0.92$)$, obesity $(\mathrm{OR}=1.27 ; 95 \% \mathrm{CI}=1.07$ to 1.49$)$, smoking $(\mathrm{OR}=1.26 ; 95 \%$ $\mathrm{CI}=1.06$ to 1.49$)$ and dyslipidemia $(\mathrm{OR}=1.35 ; 95 \% \mathrm{CI}=1.15$ to 1.57$)$ also predicted depression. Additional psychological risks were social phobia $(\mathrm{OR}=4.13 ; 95 \% \mathrm{CI}=3.45$ to 4.92$)$, type $\mathrm{D}(\mathrm{OR}=2.51 ; 95 \% \mathrm{CI}=2.16$ to 2.91$)$, loneliness $(\mathrm{OR}=2.83 ; 95 \% \mathrm{CI}=2.39$ to 3.36$)$ and life events last 12 months (per 5 events) $(\mathrm{OR}=1.74$; $95 \% \mathrm{CI}=1.53$ to 1.98$)$. $\mathrm{CVD}$ was a predictor; social support was protective $(\mathrm{OR}=0.90 ; 95 \% \mathrm{CI}=0.89$ to 0.92$)$. Partnership, alcohol abuse, hypertension, cancer, diabetes were not associated with depression.

Table 3 presents the multiple linear regression on subjective health.

As Table 3 shows, obstructive pulmonary disease and depression independently contributed to reduced subjective health in addition to sedentary behavior, smoking and comorbid somatic and mental disorders.

Subjective health was reduced in participants with obstructive pulmonary disease. In multivariable linear regression, depression was an additional factor, along with dyspnea, higher age, lower SES, a lack of a partnership, alcohol abuse, lack of active sports, obesity, smoking, type D, loneliness, lack of social support, and the presence of life events.

Supplementary Table S1 shows predictors of depression (PHQ9 $\geq 10$ ) for men and women depicted separately.

\section{Discussion}

The study presents population-based results on the association of depression, obstructive pulmonary disease (COPD or asthma) and subjective health in adults aged 35 to 74 years. In a large cohort of 15,010 participants from the community, we report data of 703 participants (4.9\%) with obstructive pulmonary disease. Multivariable logistic regression analysis were performed in order to identify associations with depression above the presence of obstructive pulmonary disease, pulmonary functional decline and dyspnea. We observed a strong association between obstructive pulmonary disease and depression. Participants with COPD or asthma are more likely to suffer from depression and reduced subjective health. Depression and obstructive pulmonary disease both independently contributed to reduced subjective health.

These findings confirm the association between obstructive diseases and depression. A meta-analytical review $^{35}$ identified that the prevalence of depressive symptoms was two times higher in people with COPD in comparison to controls. Zhang et al..$^{36}$ found significantly higher depressive symptoms in patients with COPD $(24,6 \%)$ than in controls $(11,7 \%)$. In this study, the severity of COPD was not associated with the prevalence of depression. Similarly, the meta-analysis of Atlantis et al. ${ }^{13}$ suggested that COPD elevated the risk of depression (relative risk 1.69). The outcome of COPD, e.g. COPD exacerbation, was consistently increased by depression (relative risk 1.43).

A longitudinal study by Schneider et al. $(\mathrm{n}=35,000$ patients with COPD) with a follow-up of 10 years found 16.2 cases per 1000 person-years in the COPD group compared to 9.4 cases per 1000 person-years in the control group $^{37}$. In this study, those with severe COPD were twice as likely to develop depression ${ }^{13}$ compared to patients with mild COPD. 


\begin{tabular}{|c|c|c|c|c|}
\hline & All $(N=14294)$ & No COPD/asthma $(N=13591)$ & COPD/asthma $(N=703)$ & $\mathbf{p}$ \\
\hline Age [y] & $54.8 \pm 11.1$ & $54.6 \pm 11.1$ & $57.2 \pm 10.9$ & $<0.0001$ \\
\hline Sex (\% women) & $49.6(7086)$ & $49.2(6690)$ & $56.3(396)$ & 0.00027 \\
\hline BMI $\left[\mathrm{kg} / \mathrm{m}^{2}\right]$ & $26.6(23.9 / 30.0)$ & $26.5(23.9 / 29.9)$ & $27.8(24.9 / 31.5)$ & $<0.0001$ \\
\hline \multicolumn{5}{|l|}{ Spirometry } \\
\hline FEV1 (per SD) & $297.3 \pm 77.7$ & $300.2 \pm 76.5$ & $241.8 \pm 79.9$ & $<0.0001$ \\
\hline FVC & $380 \pm 95$ & $383 \pm 95$ & $332 \pm 94$ & $<0.0001$ \\
\hline COPD/Asthma (\%) & $4.9(703)$ & $0(0)$ & $100.0(703)$ & $<0.0001$ \\
\hline NYHA $\geq 1(\%)$ & $11.9(1694)$ & $10.1(1378)$ & $45.1(316)$ & $<0.0001$ \\
\hline NYHA $\geq 2(\%)$ & $2.5(362)$ & $2.0(269)$ & $13.3(93)$ & $<\mathbf{0 . 0 0 0 1}$ \\
\hline \multicolumn{5}{|l|}{ CVRFs, comorbid diseases } \\
\hline Diabetes (\%) & $8.9(1275)$ & $8.7(1183)$ & $13.2(92)$ & 0.00013 \\
\hline Obesity (\%) & $25.1(3581)$ & $24.6(3339)$ & $34.4(242)$ & $<0.0001$ \\
\hline Smoking (\%) & $19.4(2768)$ & $19.2(2609)$ & $22.8(159)$ & 0.024 \\
\hline Hypertension (\%) & $49.3(7050)$ & $49.0(6650)$ & $56.9(400)$ & $<0.0001$ \\
\hline Dyslipidemia (\%) & $43.9(6268)$ & $43.5(5900)$ & $52.6(368)$ & $<0.0001$ \\
\hline FH of MI/Stroke (\%) & $22.1(3160)$ & $21.6(2941)$ & $31.2(219)$ & $<0.0001$ \\
\hline CVD (\%) & $11.3(1616)$ & $10.8(1473)$ & $20.3(143)$ & $<0.0001$ \\
\hline MI (\%) & $2.8(399)$ & $2.7(363)$ & $5.1(36)$ & 0.00056 \\
\hline Stroke (\%) & $1.7(246)$ & $1.6(221)$ & $3.6(25)$ & 0.00053 \\
\hline Cancer (\%) & $9.0(1281)$ & $8.7(1184)$ & $13.8(97)$ & $<0.0001$ \\
\hline \multicolumn{5}{|l|}{ Social and behavioral } \\
\hline SES & $13.00 \pm 4.43$ & $13.06 \pm 4.43$ & $11.95 \pm 4.35$ & $<0.0001$ \\
\hline Employment (\%) & $61.6(8788)$ & $62.3(8447)$ & $49.1(341)$ & $<\mathbf{0 . 0 0 0 1}$ \\
\hline Income $[€]$ & $1625.00(875.00 / 2625.00)$ & $1875.00(1125.00 / 2625.00)$ & $1375.00(875.00 / 2125.00)$ & $<0.0001$ \\
\hline Partnership (\%) & $81.3(11618)$ & $81.4(11061)$ & $79.2(557)$ & 0.15 \\
\hline Social support & $21.00(18.00 / 24.00)$ & $21.00(18.00 / 24.00)$ & $21.00(18.00 / 23.08)$ & 0.0067 \\
\hline Loneliness (\%) & $10.5(1493)$ & $10.3(1393)$ & $14.3(100)$ & 0.0012 \\
\hline Alcohol (gram/day) & $5.03(0 / 17.60)$ & $5.03(0 / 17.60)$ & $2.10(0 / 14.07)$ & 0.0088 \\
\hline Active sports (\%) & $49.0(7008)$ & $49.3(6696)$ & $44.4(312)$ & 0.012 \\
\hline Subjective health & $67.11 \pm 19.85$ & $67.73 \pm 19.48$ & $55.19 \pm 22.83$ & $<0.0001$ \\
\hline Ex-smoker (\%) & $34.9(4983)$ & $35.0(4749)$ & $33.5(234)$ & 0.46 \\
\hline Passive smoker (\%) & $3.7(528)$ & $3.7(508)$ & $2.8(20)$ & 0.26 \\
\hline Pack years & $0.09(0 / 3.21)$ & $0.09(0 / 3.14)$ & $0.14(0 / 5.66)$ & 0.012 \\
\hline \multicolumn{5}{|l|}{ Mental } \\
\hline PHQ9 $\geq 10(\%)$ & $8.0(1139)$ & $7.5(1025)$ & $16.2(114)$ & $<0.0001$ \\
\hline Suicidal thoughts (PHQ9) (\%) & $7.6(1084)$ & 7.5 (1006) & $11.2(78)$ & 0.00058 \\
\hline History of depression (\%) & $12.0(1707)$ & $11.4(1551)$ & $22.2(156)$ & $<0.0001$ \\
\hline Panic (\%) & $4.8(662)$ & $4.5(595)$ & $9.8(67)$ & $<0.0001$ \\
\hline History of anxiety disorder (\%) & $7.2(1031)$ & $6.9(942)$ & $12.7(89)$ & $<0.0001$ \\
\hline Social Phobia (Mini-Spin $\geq 6)(\%)$ & $7.3(1044)$ & $7.3(982)$ & $8.9(62)$ & 0.12 \\
\hline GAD2 $\geq 3(\%)$ & $6.6(936)$ & $6.3(846)$ & $12.9(90)$ & $<0.0001$ \\
\hline Type D (\%) & $23.7(3377)$ & $23.5(3181)$ & $28.0(196)$ & 0.0071 \\
\hline \multicolumn{5}{|l|}{ PHQ9 classes } \\
\hline None (\%) & $64.7(9183)$ & $65.3(8823)$ & $51.6(360)$ & n.a. \\
\hline Minimal (\%) & $27.4(3891)$ & $27.2(3667)$ & $32.1(224)$ & n.a. \\
\hline Mild (\%) & $6.0(856)$ & $5.7(769)$ & $12.5(87)$ & n.a. \\
\hline Moderately (\%) & $1.5(210)$ & $1.4(187)$ & $3.3(23)$ & n.a. \\
\hline Severe (\%) & $0.5(64)$ & $0.4(60)$ & $0.6(4)$ & n.a. \\
\hline \multicolumn{5}{|l|}{ Medication } \\
\hline Antidepressant (\%) & $5.6(790)$ & $5.3(712)$ & $11.1(78)$ & $<0.0001$ \\
\hline SSRI (\%) & $1.9(272)$ & $1.8(244)$ & $4.0(28)$ & 0.00031 \\
\hline Anxiolytic (\%) & $0.9(125)$ & $0.8(110)$ & $2.1(15)$ & 0.0013 \\
\hline Benzodiazepine (\%) & $0.9(121)$ & $0.8(107)$ & $2.0(14)$ & 0.0026 \\
\hline
\end{tabular}

Table 1. Demographical and clinical characteristics of participants with and without medicated COPD/ asthma. Presented are means and standard deviations, rep. percentages; 1) median; Interquartile range; $\mathrm{CVD}=$ cardiovascular disease $\mathrm{MI}=$ myocardial infarction; $\mathrm{FH}=$ family history; $\mathrm{SES}=$ socioeconomic status; PHQ9 = Patient Health Questionnaire Depression Score; GAD2 = Generalized Anxiety Score; SSRI = Selective Serotonin Reuptake Inhibitor. 


\begin{tabular}{|c|c|c|c|}
\hline & \multicolumn{3}{|c|}{$(N=13875, N=1078$ events $)$} \\
\hline & OR & $95 \% \mathrm{CI}(\mathrm{L}, \mathrm{U})$ & $\mathbf{P}$ \\
\hline Age $[5 y]$ & 0.96 & $0.92,1.01$ & 0.17 \\
\hline Sex (\% women) & 1.72 & $1.41,2.11$ & $<0.0001$ \\
\hline \multicolumn{4}{|l|}{ Spirometry } \\
\hline FEV1 (per SD) & 1.17 & $1.05,1.32$ & 0.0055 \\
\hline COPD/asthma (\%) & 1.71 & $1.30,2.23$ & 0.00010 \\
\hline NAHA $\geq 1(\%)$ & 2.19 & $1.81,2.64$ & $<0.0001$ \\
\hline \multicolumn{4}{|l|}{ CVRFs, comorbid diseases } \\
\hline Diabetes (\%) & 1.14 & $0.89,1.45$ & 0.29 \\
\hline Obesity (\%) & 1.26 & $1.06,1.49$ & 0.0063 \\
\hline Smoking (\%) & 1.25 & $1.05,1.48$ & 0.0087 \\
\hline Hypertension (\%) & 0.99 & $0.84,1.17$ & 0.97 \\
\hline Dyslipidemia (\%) & 1.34 & $1.15,1.57$ & 0.00016 \\
\hline CVD (\%) & 1.30 & $1.03,1.62$ & 0.020 \\
\hline Cancer (\%) & 0.95 & $0.73,1.22$ & 0.70 \\
\hline \multicolumn{4}{|l|}{ Social and behavioral } \\
\hline SES & 0.98 & $0.96,0.99$ & 0.034 \\
\hline Partnership (\%) & 1.04 & $0.87,1.24$ & 0.63 \\
\hline Social support & 0.90 & $0.88,0.91$ & $<0.0001$ \\
\hline Loneliness (\%) & 2.83 & $2.38,3.35$ & $<0.0001$ \\
\hline Alcohol abuse & 0.75 & $0.46,1.17$ & 0.24 \\
\hline Active sports (\%) & 0.79 & $0.68,0.91$ & 0.0019 \\
\hline \multicolumn{4}{|l|}{ Mental } \\
\hline Social Phobia (Mini-Spin $\geq 6)(\%)$ & 4.12 & $3.45,4.92$ & $<0.0001$ \\
\hline Type D (\%) & 2.50 & $2.15,2.91$ & $<0.0001$ \\
\hline Life events last 12 months (per 5 events) & 1.74 & $1.53,1.97$ & $<0.0001$ \\
\hline
\end{tabular}

Table 2. Multivariable logistic regression model: predictors of depression (PHQ9 $\geq 10$ ). Note: $95 \%$ CI [L, $\mathrm{U}]=95 \%$ confidence interval [lower value, upper value]. p: p-value. Depression: (PHQ-9 $\geq 10$ ). Social Phobia: Mini-Spin $\geq 6$. Life events last 12 months: per 5 events.

The high comorbidity could be explained with symptoms present in COPD and depression. Both disorders are associated with withdrawal, anhedonia, fatigue, poor appetite, sleep disturbance, loss of concentrations and energy and reduced physical activity ${ }^{38}$.

However, in patients with COPD or asthma, the impact of social, biological, behavioral and psychological factors has not been thoroughly elucidated to date. It is important to clarify the impact of these factors on the risk of developing depression and reduced subjective health.

Smoking has long been known as an important cause of COPD in developed countries ${ }^{6}$. A positive association between smoking and depression has been reported ${ }^{19,39-41}$. Melvyn et al. ${ }^{35}$ reported that smoking status was not a significant moderator in predicting prevalence of depressive symptoms. This is not in line with the findings of this study. We found an association between smoking and prevalence of depression in participants with COPD or asthma. However, when analyzed separately for women and men we only found a significant association in women, but not in men (Supplementary Table S1).

Mevelyn et al..$^{35}$ found that sex was not a significant moderator. Hanian et al. ${ }^{9}$ reported a significantly higher prevalence of depression in women suffering from COPD. In multivariable logistic regression analysis, we found an association between sex and prevalence of depression in participants with COPD or asthma.

Ottenheim et al. ${ }^{42}$ found in the oldest subjects, that COPD independently contributed to an increased risk of developing depressive symptoms. On the other hand, there is evidence, that age is not associated with higher depression prevalence in patients with $\mathrm{COPD}^{7}$. In this study, we found that participants suffering from obstructive pulmonary disease were older. In multiple regression analysis, however, age was not associated with higher depression prevalence in patients with COPD.

Findings of this study resonate with previous studies showing that the relationship between COPD or asthma and depression is indeed complex. Especially the degree of subjective impairment (dyspnea) played a significant adverse role, compounded by reduced objective function, social disadvantage, unhealthy behavior (smoking, no active sports, obesity, dyslipidemia), comorbid CVD, mental comorbidity (type D, social phobia), a lack of social support and loneliness and additional adverse life events in the previous years.

As we had expected, subjective health was also reduced in participants with obstructive pulmonary disease. In multivariable linear regression, depression was an additional factor, along with dyspnea, higher age, lower SES, a lack of a partnership, alcohol abuse, lack of active sports, obesity, smoking, type D, loneliness, lack of social support, and the presence of life events.

The complex interplay of biological, psychosocial and behavioral risk factors should be taken into account in the clinical care and guidance of COPD and asthma patients. In addition to the well-known risk profile of 


\begin{tabular}{|c|c|c|c|}
\hline & Estimate & $95 \% \mathrm{CI}[\mathrm{L}, \mathrm{U}]$ & $\mathbf{P}$ \\
\hline Age [5y] & -0.40 & $-0.57,-0.23$ & $<0.0001$ \\
\hline Sex (\% women) & -0.12 & $-0.77,0.51$ & 0.70 \\
\hline \multicolumn{4}{|l|}{ Spirometry } \\
\hline COPD/asthma (\%) & -4.74 & $-6.30,-3.17$ & $<0.0001$ \\
\hline NYHA $\geq 1(\%)$ & -9.07 & $-10.1,-8.04$ & $<0.0001$ \\
\hline \multicolumn{4}{|l|}{ CVRFs, comorbid diseases } \\
\hline Diabetes (\%) & -3.80 & $-4.94,-2.67$ & $<0.0001$ \\
\hline Obesity (\%) & -4.37 & $-5.12,-3.61$ & $<0.0001$ \\
\hline Smoking (\%) & -2.68 & $-3.49,-1.88$ & $<0.0001$ \\
\hline Hypertension (\%) & -3.36 & $-4.05,-2.68$ & $<0.0001$ \\
\hline Dyslipidemia (\%) & -2.44 & $-3.10,-1.77$ & $<0.0001$ \\
\hline CVD (\%) & -7.06 & $-8.08,-6.03$ & $<0.0001$ \\
\hline Cancer (\%) & -4.52 & $-5.58,-3.45$ & $<0.0001$ \\
\hline \multicolumn{4}{|l|}{ Social and behavioral } \\
\hline SES & 0.29 & $0.21,0.36$ & $<0.0001$ \\
\hline Partnership (\%) & -1.04 & $-1.88,-0.20$ & 0.015 \\
\hline Social support & 0.47 & $0.37,0.56$ & $<0.0001$ \\
\hline Loneliness (\%) & -3.97 & $-5.07,-2.86$ & $<0.0001$ \\
\hline Alcohol abuse & -2.03 & $-3.83,-0.23$ & 0.027 \\
\hline Active sports (\%) & 2.13 & $1.51,2.76$ & $<0.0001$ \\
\hline \multicolumn{4}{|l|}{ Mental } \\
\hline Depression (\%) & -13.3 & $-14.6,-12.0$ & $<0.0001$ \\
\hline COPD/Asthma \& Depression (\%) & -19.9 & $-23.4,-16.4$ & $<0.0001$ \\
\hline Social Phobia (\%) & -0.94 & $-2.19,0.30$ & 0.14 \\
\hline Type D (\%) & -3.93 & $-4.69,-3.17$ & $<0.0001$ \\
\hline Life events last 12 months & -1.23 & $-1.86,-0.59$ & 0.00014 \\
\hline
\end{tabular}

Table 3. Linear regression on subjective health (overall) $(N=12267)$. Note: $95 \% \mathrm{CI}[\mathrm{L}, \mathrm{U}]=95 \%$ confidence interval [lower value, upper value]. p: p-value. Depression: (PHQ-9 $\geq 10$ ). Social Phobia: Mini-Spin $\geq 6$. Life events last 12 months: per 5 events.

comorbid medical conditions and distress, a pattern of social disadvantage (lower SES) and behavioral risks needs to be attended in medical care. Promoting health behavior such as smoking cessation, adequate exercising and reducing weight may not only improve the somatic course of the disease but also reduce detrimental mental health consequences. Additionally, promotion of social support and integration may provide additional avenues of intervention.

Strengths and limitations. Strengths refer to the large representative community sample and the comprehensive assessment of multiple sources integrating demographic, psychosocial, and behavioral determinants with mental and somatic comorbidities. The analyses covered participants who reported they were diagnosed and treated with COPD or asthma by a physician (and not limited to those who seek health care as in most previous studies). Yet, we were able to include a comprehensive range of biological variables including pulmonary function and symptoms (dyspnea) and comorbid disorders, social and psychological variables.

Limitations pertain to the fact that the medical diagnosis was self-reported, however, the functional measures show that there was significant objective and subjective impairment. Future analyses will test these findings in participants who are unaware of their obstructive pulmonary disease.

There is still a considerable need for prospective studies to continue investigation into the causality and temporal relationship between obstructive pulmonary disease and depressive symptoms. Participants with less severe complaints are more likely to take part in a community study. This might have led to selection bias towards oversampling those with the less severe depressive, respectively pulmonary symptoms. Also, we relied on data of validated questionnaires. We could not use expert clinical ratings of depression. Young ( $<35$ years) and older participants ( $>75$ years) did not take part in the examination. Prospective work should also target effective interventions (e.g. lifestyle, psychotherapy) to prevent and reduce depression in patients with COPD or asthma.

\section{Conclusion}

Physical and mental health are strongly interrelated in medicated chronic obstructive pulmonary disease. There has been a strong increase of depression $(16.2 \%$ vs. $7.5 \%)$ compared to participants without obstructive pulmonary disease. Complex underlying factors include sociodemographic (female sex, low SES), medical (FEV1, dyspnea), cardiovascular risk factors (smoking, obesity, dyslipidemia), and psychosocial factors (social phobia, type D, low social support, loneliness and stressful life events). The presence of obstructive lung disease and depression independently contributed to reduced subjective health. Promoting health behavior and social integration may not only improve the somatic course of the disease, but also mental health ${ }^{43}$. 


\section{Data availability}

For approved reasons, some access restrictions apply to the data underlying these findings. Data sets contain identifying participant information, which is not suitable for public deposition. Access to the local database is available upon request to the corresponding author.

Received: 22 April 2019; Accepted: 3 December 2019;

Published online: 27 December 2019

\section{References}

1. Postma, D. S. \& Timens, W. Remodeling in Asthma and Chronic Obstructive Pulmonary Disease. Proc. Am. Thorac. Soc. 3, 434-439 (2006).

2. Fabbri, L. M., Beghe, B. \& Agusti, A. COPD and the solar system: introducing the chronic obstructive pulmonary disease comorbidome. Am. J. Resp. Crit. Care Med. 186, 117-119 (2012).

3. Negewo, N. A., McDonald, V. M. \& Gibson, P. G. Comorbidity in chronic obstructive pulmonary disease. Respir. Investig. 53, 249-258 (2015)

4. Decramer, M., Janssens, W. \& Miravitlles, M. Chronic obstructive pulmonary disease. Lancet 379, 1341-1351 (2012).

5. Divo, M. et al. Comorbidities and risk of mortality in patients with chronic obstructive pulmonary disease. Am. J. Respir. Crit. Care. Med. 186, 155-161 (2012).

6. Mathew, A. R., Yount, S. E., Kalhan, R. \& Hitsman, B. Psychological Functioning in Patients with Chronic Obstructive Pulmonary Disease: A Preliminary Study of Relations with Smoking Status and Disease Impact. Nicotine Tob. Res. 21, 686-690 (2019).

7. Di Marco, F. et al. Anxiety and depression in COPD patients: the roles of gender and disease severity. Respir. Med. 100, 1767-1774 (2006).

8. Miravitlles, M. et al. Factors associated with depression and severe depression in patients with COPD. Respir. Med. 108, 1615-1625 (2014).

9. Hanania, N. A. et al. Determinants of depression in the ECLIPSE chronic obstructive pulmonary disease cohort. Am. J. Respir. Crit. Care Med. 183, 604-611 (2011).

10. Papaioannou, A. I. et al. The impact of depressive symptoms on recovery and outcomes of hospitalised COPD exacerbations. Eur. Respir. J. 41, 815-823 (2013).

11. Xu, W. et al. Independent effect of depression and anxiety on chronic obstructive pulmonary disease exacerbations and hospitalizations. Am. J. Respir. Crit. Care Med. 178, 913-920 (2008).

12. Garcia-Polo, C. et al. Factors associated with high resource utilisation among COPD patients. Respir. Med. 106, 1734-1742 (2012).

13. Atlantis, E., Fahey, P., Belinda Cochrane, B. \& Smith, S. Bidirectional associations between clinically relevant depression or anxiety and chronic obstructive pulmonary disease (COPD): a systematic review and meta-analysis. Chest 144, 766-777 (2013).

14. De Voogd, J. N. et al. Depressive symptoms as predictors of mortality in patients with COPD. Chest 135, 619-625 (2009).

15. Ford, E. S. et al. Self-reported asthma and health-related quality of life: findings from the behavioral risk factor surveillance system. Chest 123, 119-127 (2003).

16. Opolski, M. \& Wilson, I. Asthma and depression: a pragmatic review of the literature and recommendations for future research. Clin. Pract. Epidemiol. Ment. Health. 1, 18 (2005).

17. Papava, I. et al. The impact of coping on the somatic and mental status of patients with COPD: a cross-sectional study. Int. J. Chron. Obstruct. Pulmon. Dis. 11, 1343-1351 (2016).

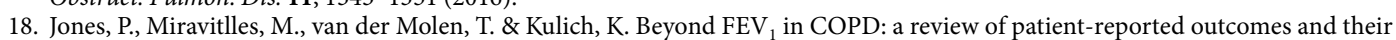
measurement. Int. J. Chron. Obstruct. Pulmon. Dis. 7, 697-709 (2012).

19. Michal, M. et al. Association of mental distress with smoking status in the community: Results from the Gutenberg Health Study. J. Affect. Disord. 146, 355-360 (2013).

20. Beutel, M. E. et al. New onset of depression in aging women and men: contributions of social, psychological, behavioral, and somatic predictors in the community. Psychol. Med. 49, 1148-1155 (2019).

21. Lehrer, P. M., Feldman, J., Giardino, N., Song, H. S. \& Schmaling, K. Psychological aspects of asthma. J Consult Clin. Psychol. 70, 691-711 (2002).

22. Ritz, T. \& Steptoe, A. Emotion and pulmonary function in asthma: reactivity in the field and relationship with laboratory induction of emotion. Psychosom. Med. 62, 808-815 (2000).

23. Höhn, R. et al. The Ophthalmic Branch of the Gutenberg Health Study: Study Design, Cohort Profile and Self-Reported Diseases. PLOS ONE 10 (2015).

24. Wild, P. et al. The Gutenberg Health Study. Bundesgesundheitsblatt, Gesundheitsforschung, Gesundheitsschutz 55, 824-829 (2012).

25. Lowe, B. et al. Diagnosing ICD-10 depressive episodes: superior criterion validity of the Patient Health Questionnaire. Psychotherapy and Psychosomatics 73, 386-390 (2004).

26. Kroenke, K., Spitzer, R. L., Williams, J. B., Monahan, P. O. \& Lowe, B. Anxiety disorders in primary care: prevalence, impairment, comorbidity, and detection. Ann. Intern. Med. 146, 317-325 (2007).

27. Lowe, B. et al. Detecting panic disorder in medical and psychosomatic outpatients: comparative validation of the Hospital Anxiety and Depression Scale, the Patient Health Questionnaire, a screening question, and physicians' diagnosis. J. Psychosom. Res. 55, 515-519 (2003).

28. Wiltink, J. et al. Mini-Social Phobia Inventory (mini-SPIN): psychometric properties and population based norms of the German version. Psychosom. Med. Psychother. 63, 115-115 (2017).

29. Denollet, J. DS14: standard assessment of negative affectivity, social inhibition, and Type D personality. Psychosom. Med. 67, 89-97 (2005).

30. Lampert, T. \& Kroll, L.E. Die Messung des sozioökonomischen Status in sozialepidemiologischen Studien. In Gesundheitliche Ungleichheit. (ed. Richter, M., Hurrelmann, K.) 309-334 (Verlag für Sozialwissenschaften 2009).

31. Beutel, M. E. et al. Loneliness in the general population: prevalence, determinants and relations to mental health. BMC Psychiatry 17, 97 (2017).

32. Peters, T. et al. Validity of a short questionnaire to assess physical activity in 10 European countries. Eur. J. Epidem. 27, 15-25 (2012).

33. Wiltink, J. et al. Associations between depression and different measures of obesity (BMI, WC, WHtR, WHR). BMC Psychiatry 13, 223 (2013).

34. Magnussen, C. et al. FEV1 and FVC predict all-cause mortality independent of cardiac function - Results from the population-based Gutenberg Health Study. Int. J. Cardiol. 234, 64-68 (2017).

35. Zhang, M. W., Ho, R. C., Cheung, M. W., Fu, E. \& Mak, A. Prevalence of depressive symptoms in patients with chronic obstructive pulmonary disease: a systematic review, meta-analysis and meta-regression. Gen. Hosp. Psychiatry 33, 217-223 (2011).

36. Zhang, M. W. et al. Prevalence of depressive symptoms in patients with chronic obstructive pulmonary disease: a systematic review, meta-analysis and meta-regression. Gen. Hosp. Psychiatry 33, 217-223 (2011)

37. Schneider, C., Jick, S. S., Bothner, U. \& Meier, C. R. COPD and the risk of depression. Chest 137, 341-7 (2010). 
38. Yohannes, A. M. et al. Depression and anxiety in chronic heart failure and chronic obstructive pulmonary disease: prevalence, relevance, clinical implications and management principles. Int. J. Geriatr. Psychiatry 25, 1209-1221 (2010).

39. Fluharty, M. et al. The Association of Cigarette Smoking With Depression and Anxiety: A Systematic Review. Nicotine tob. Res. 19, 3-13 (2016).

40. Quellette, D. R. \& Lavoie, K. L. Recognition, diagnosis, and treatment of cognitive and psychiatric disorders in patients with COPD. Int. J. Chron. Obstruc. Pulmon. Dis. 12, 639-650 (2017)

41. Wong, S. T. et al. The diagnosis of depression and its treatment in Canadian primary care practices: an epidemiological study. CMAJ Open 2, 337-342 (2014).

42. Ottenheim, N. R., Giltay, E. J., Zitman, F. G., van der Mast, R. C. \& de Craen, A. J. Risk of depressive symptoms in the oldest old subjects with chronic obstructive pulmonary disease: the Leiden 85-plus study. Am. J. Geriatr. Psychiatry 18, 388-94 (2010).

43. Miravitlles, M. \& Ribera, A. Understanding the impact of symptoms on the burden of COPD. Resp. Res. 18, 67 (2017).

\section{Acknowledgements}

We express our gratitude to the study participants and staff of the Gutenberg Health Study. The Gutenberg Health Study is funded through the government of Rhineland-Palatinate ("Stiftung Rheinland-Pfalz für Innovation", contract AZ 961-386261/733), the research programs "Wissen schafft Zukunft" and "Center for Translational Vascular Biology (CTVB)" of the Johannes Gutenberg- University of Mainz, and its contract with Boehringer Ingelheim and PHILIPS Medical Systems, including an unrestricted grant for the Gutenberg Health Study. The funders had no role in study design, data collection and analysis, decision to publish, or preparation of the manuscript.

\section{Author contributions}

All authors have made substantial contributions to this work, and all have been involved in drafting or revising the manuscript. Conceived and designed the studies: Prof Beutel, Dr. Ghaemi Kerahrodi, Mr. Schulz, Prof. Brähler, Prof. Münzel, Prof. Lackner, Prof. Pfeiffer, Prof. Michal and Dr. Wiltink. Analyzed data: Mr. Schulz. and Mr. Toenges. Contributed to interpretation of data: Dr. Ghaemi Kerahrodi, Prof. Beutel and Prof. Brähler. Drafted the article: Dr. Ghaemi Kerahrodi, Prof Beutel and Prof. Brähler. Revised the article critically: Prof. Wild, Prof. Münzel, Prof. Lackner, Prof. Pfeiffer, Prof. Michal, Dr. Wiltink and Mr. Schulz. All authors have read and approved the final manuscript as submitted.

\section{Competing interests}

P.S. Wild reports grants and personal fees from Boehringer Ingelheim, grants from Philips Medical Systems, grants and personal fees from Sanofi-Aventis, grants and personal fees from Bayer Vital, grants from Daiichi Sankyo Europe, personal fees from Bayer Health Care, personal fees from Astra Zeneca, personal fees and non-financial support from DiaSorin, non-financial support from I.E.M. P.S. Wild and T. Münzel are PIs of the German Center for Cardiovascular Research (DZHK). The other authors state that they have no conflict of interest and no competing interests.

\section{Additional information}

Supplementary information is available for this paper at https://doi.org/10.1038/s41598-019-56440-9.

Correspondence and requests for materials should be addressed to J.G.K.

Reprints and permissions information is available at www.nature.com/reprints.

Publisher's note Springer Nature remains neutral with regard to jurisdictional claims in published maps and institutional affiliations.

(c) (i) Open Access This article is licensed under a Creative Commons Attribution 4.0 International

License, which permits use, sharing, adaptation, distribution and reproduction in any medium or format, as long as you give appropriate credit to the original author(s) and the source, provide a link to the Creative Commons license, and indicate if changes were made. The images or other third party material in this article are included in the article's Creative Commons license, unless indicated otherwise in a credit line to the material. If material is not included in the article's Creative Commons license and your intended use is not permitted by statutory regulation or exceeds the permitted use, you will need to obtain permission directly from the copyright holder. To view a copy of this license, visit http://creativecommons.org/licenses/by/4.0/.

(c) The Author(s) 2019 